\title{
DEMOGRAFIA E O ENVELHECIMENTO EM PORTUGAL E NO BRA- SIL: REFLEXÕES PARA O DESENVOLVIMENTO REGIONAL
}

\author{
DEMOGRAPHY AND AGING IN PORTUGAL AND BRAZIL: REFLECTIONS FOR REGIONAL \\ DEVELOPMENT
}

\begin{abstract}
Miriam Cabrera Corvelo Delboni', Silvia Virginia Coutinho Areosa², Juliana Rohde', Aline Sarturi Ponte ${ }^{4}$, Nathalie da Costa Nascimento ${ }^{5}$, Andreisi Carbone Anversa ${ }^{6}$
\end{abstract}

RECEBIDO EM: 30/08/2018 | ACEITO EM:26/06/2019

DOI: $10.5902 / 2317175834555$

\section{RESUMO}

O crescimento demográfico mundial de idosos exige preparação dos países para atuarem nos impactos decorrentes desse aumento e longevidade populacional. O desafio que está posto diz respeito a oferta de cuidados em saúde para a população que envelhece e sustentação econômica dos serviços em cada região. Com o objetivo de compreender as mudanças demográficas ocorridas em Portugal e no Brasil, no que se refere ao envelhecimento populacional, realizou-se um levantamento de dados secundários. Estes foram selecionados a partir dos dados estatísticos censitários e organismos oficiais, tais como: Instituto Nacional de Estatística (INE, 2015), Censo (INE, 2011), Direção Geral do Ministério da Saúde, Câmara Municipal de Braga, Ministério da Saúde do Brasil e Secretaria Estadual de Saúde do Rio Grande do Sul. Dessa forma, apresenta-se a relação do envelhecimento populacional com o desenvolvimento regional nos dois países. Os dados demonstram que o envelhecimento é uma realidade em Portugal e no Brasil e em ambos a tendência é alcançar índices cada vez maiores nas próximas décadas. Conclui-se que em todas as regiões dos países estudados há um envelhecimento populacional evidente, no entanto, com particularidades distintas para cada nação.

Palavras-chave: Envelhecimento. Demografia. Desenvolvimento Regional

1 Graduada em Terapia Ocupacional pela Pontifícia Universidade Católica de Campinas, Especialista em Terapia da Mão e Mestrado em Reabilitação pela Universidade Federal de São Paulo. Doutora em Desenvolvimento Regional. Atualmente é professora do Departamento de Terapia Ocupacional da Universidade Federal de Santa Maria (UFSM) e Docente da Pós-Graduação Reabilitação Físico Motora e do Programa de Mestrado em Gerontologia da UFSM

2 Graduada em Psicologia pela Universidade do Vale do Rio dos Sinos, mestrado em Psicologia Social e da Personalidade pela Pontifícia Universidade Católica do Rio Grande do Sul e doutorado sanduíche em Servico Social pela Pontifícia Universidade Católica do Rio Grande do Sul. Pós Doutorado em Serviço Social pela PUCRS. Atualmente é professora do Curso de Psicologia e do Programa de Mestrado e Doutorado em Desenvolvimento Regional. Docente e Coordenadora do Mestrado Profissional em Psicologia da Universidade de Santa Cruz do Sul- UNISC

3 Graduada em Psicologia pela Universidade de Santa Cruz do Sul. Residente no Hospital Santa Cruz - HSC, pelo Programa de Residência Multiprofissional em Saúde - Intensivismo, urgência e emergência. Integrante do Grupo de estudos e pesquisas em envelhecimento e cidadania.

4 Graduada em Terapeuta Ocupacional pelo Centro Universitário Franciscano. Especialista em Reabilitação Físico Motora com ênfase nas desordens do movimento humano pela Universidade Federal de Santa Maria. Mestre e doutoranda em Distúrbios da Comunicação Humana pela Universidade Federal de Santa Maria.

5 Graduada em Terapeuta Ocupacional graduada pela Universidade Federal de Santa Maria (UFSM). Atualmen-

te, residente no Programa de Residência Multiprofissional Integrada em Gestão e Atenção Hospitalar no Sistema Público de Saúde com ênfase na Hemato-Oncologia - UFSM e mestranda do PPG em Gerontologia - UFSM.

6 Graduada em Terapeuta Ocupacional formada pela Universidade Federal de Santa Maria. Mestre em Distúrbios da Comunicação Humana/UFSM. 
MIRIAM CABRERA CORVELO DELBONI, SILVIA VIRGINIA COUTINHO AREOSA, JULIANA ROHDE, ALINE SARTURI PONTE, NATHALIE DA COSTA NASCIMENTO, ANDREISI CARBONE ANVERSA

\begin{abstract}
The global demographic growth of the elderly requires the preparation of the countries to act on the impacts resulting from this increase and population longevity. The challenge that is posed concerns the provision of health care for the aging population and the economic support of services in each region. In order to understand the demographic changes that occurred in Portugal and Brazil, with regard specifically to population aging, a survey of secondary data was carried out. The secondary data used were statistical census data and official bodies, such as: National Institute of Statistics (INE, 2015), Census (INE, 2011), General Directorate of the Ministry of Health, Braga Municipal Council, Ministry of Health of Brazil and State Health Secretariat of Rio Grande do Sul. Thus, the relation between population aging and regional development in both countries. The data show that aging is a reality in Portugal and Brazil, and both tend to reach ever higher rates in the coming decades. It is concluded that in all the regions of the studied countries there is an evident population aging, however with distinct peculiarities for each region and country.
\end{abstract}

Keywords: Aging. Demography. Regional development

\title{
1 Introdução
}

O envelhecimento populacional é um fenômeno complexo, irreversível e prioritário na agenda dos governos, necessitando de intervenções que desafiam o Estado e a própria sociedade a desenvolverem políticas públicas para atender os direitos, as demandas e necessidades desse segmento. $\mathrm{O}$ crescimento demográfico mundial da população idosa exige a preparação adequada dos países para atender às múltiplas demandas sociais, previdenciárias, de saúde e cuidados (ALCÂNTARA et al., 2016). As mudanças ocorridas na fecundidade e na mortalidade registradas em escala internacional resultaram na desaceleração do crescimento da população e no envelhecimento progressivo da estrutura etária (Carvalho, 2015).

Para compreender o envelhecer, é necessário estabelecer alguns conceitos pautadores, como o do envelhecimento, o da velhice e o da pessoa idosa. Para Gardner (2006), o envelhecimento refere-se ao processo de desenvolvimento humano, que compreende desde a concepção até à morte. A velhice refere-se à última fase do ciclo de vida, logo, as pessoas que se encontram nesse período são consideradas idosas. $O$ envelhecimento é um processo pessoal, natural e singular de cada pessoa, e, junto com a expectativa de vida, representam importantes indicadores sociais, que apontam, inclusive, o nível de desenvolvimento de uma região, estado e país. Em diversas sociedades, a velhice é compreendida como uma fase de doenças e dores e tende a ser vista como uma fase de desvalorização, mantendo a visão errônea sobre o que é ser idoso na atualidade (AREOSA, 2012). Nessa perspectiva, a velhice está no imaginário social como uma carga econômica, seja para a família ou para a sociedade (MINAYO, 2002). Entretanto, essa representação social tem se modificado ao longo das últimas décadas, provavelmente em virtude das Diretrizes 
Internacionais e de outras contribuições multidisciplinares para o avanço desse paradigma, as quais dão ênfase à participação ativa do idoso na sociedade e no desenvolvimento desta (KIST; AREOSA, 2014).

Sen (2000) reflete sobre as questões do desenvolvimento como sendo um processo de expansão das liberdades individuais, que seriam o principal meio e fim do desenvolvimento. Visto dessa forma, para se alcançar tais liberdades seria preciso atenuar as causas das desigualdades sociais e proporcionar, a toda a população, o acesso à qualidade de saúde, educação, trabalho, lazer, bem como um meio ambiente saudável. Isso significa dizer que é preciso oportunizar a todos a participação em um ambiente democrático, igualitário e justo. Para que haja desenvolvimento, deve-se considerar o capital humano (conhecimentos, habilidades e competências da população local); o capital social (organização social e empoderamento da população local); a governança (maneiras de liderar, coordenar, intervir, participar e negociar conflitos nos processos decisórios locais) e o uso sustentável do capital natural (BECKER, 2000). Para o autor, o conceito de desenvolvimento - local ou regional - só pode ser completo quando há valorização da qualidade de vida dos indivíduos, da participação social e do bem-estar das gerações presentes e futuras. Esse conceito busca a melhoria da vida dos atores individual e coletivamente, pela inserção da comunidade no âmbito global e pelo reconhecimento das particularidades próprias da região em que ela está inserida, ressaltando a identidade e a cooperação.

Para Minayo (2002), a questão do envelhecimento outrora era tratada como questão da vida privada, ou como assunto de caridade pública, no caso dos pobres e indigentes, ou, ainda, apenas como uma questão médica. Mesmo com todos os avanços, essa visão ocorre nas práticas sociais de cuidado com os idosos, inscritas culturalmente nas sociedades ocidentais. Uma das grandes mudanças na organização social centra-se nas famílias, por ter havido profundas transformações em seu desenho demográfico, composição familiar, ambientes e formas de vida doméstica, bem como na organização do trabalho, ao mesmo tempo em que houve uma acelerada urbanização e concentração de polos urbanos. Desse modo, o tema do envelhecimento se articula ao do Desenvolvimento Regional, já que a longevidade é uma das maiores conquistas de um país, estado ou região. Contudo, o processo de envelhecer pode ocorrer de diferentes maneiras em cada lugar, dependendo das condições regionais presentes (KIST; AREOSA, 2014), já que as características econômicas, sociais, culturais e políticas distintas em cada país, estado e região, são determinantes (SILVEIRA; FELLIPPI; CAMPOS, 2013).

Dados apresentados pela ONU (2002) apontavam que, na época, 64\% dos idosos viviam em regiões menos desenvolvidas. Já em 2050, a estimativa é que esse número se aproxime de $80 \%$ (MORAES, 2012; CARVALHO, 2015). Nesse contexto, é necessário que haja dedicação para que se reduzam as desigualdades sociais, pela diminuição da exclusão e do preconceito relacionado ao idoso. 
Ademais, é determinante que esse indivíduo possa exercer a sua cidadania e que seja compreendido como mais um ator que contribui para o desenvolvimento de sua própria vida e da sociedade em que está inserido (BERNARDES, 2007). Dessa forma, o problema de pesquisa destacado apoia-se na necessidade da compreensão da relação demográfica para o desenvolvimento regional no que cerne os apontamentos de políticas públicas, quer sejam de saúde ou sociais, direcionadas, no caso em questão, aos idosos. Escolheu-se os dados censitários de Portugal e do Brasil para a reflexão, visto que o país lusitano já fez a transição demográfica enquanto no Brasil vivencia-se essa transformação rapidamente (ALCÂNTRA; CAMARANO; GIACOMIM, 2016). Logo, o objetivo do presente artigo é traçar alguns paralelos entre as mudanças demográficas ocorridas em Portugal e no Brasil, no que se refere especificamente ao envelhecimento populacional. Apresentam-se os dados relativos a esta temática, em ambos os países e suas regiões correspondentes, de forma a contribuir com a reflexão da temática do envelhecimento para o desenvolvimento regional.

\section{Método de pesquisa}

\subsection{As fontes de informação:}

Adotou-se a abordagem qualitativa, utilizando a ferramenta do estudo documental de fontes secundárias de Portugal e Brasil buscando identificar dados censitários nos documentos pesquisados. Trata-se de uma elaboração de temática transversal, marcando o primeiro tratamento dos dados brutos para um posterior corpus temático (MINAYO, 2010).

A pesquisa baseada em documentos contribuiu para identificar e construir os significados e interpretações, fortalecendo com informações factuais, as questões de interesse do estudo (FLICK, 2010).

\subsection{Procedimento de Produção dos dados}

Os primeiros documentos elencados para a produção dos dados foram as informações censitárias de cada país. Em Portugal os dados foram adquiridos através do Instituo Nacional de Estatística (INE) e no Brasil os dados censitários do Instituto Brasileiro de Geografia e Estatística (IBGE). Além disso, houve busca ativa de documentos, pesquisados pelas seguintes categorias: demografia, análise demográfica, distribuição da população, distribuição populacional, inquéritos demográficos, levantamento demográfico, pesquisa demográfica.

\subsection{Critérios de Análise de dados}

Para a pré análise, os documentos a serem analisados foram escolhidos a partir de uma busca ativa em ambos os países no ano de 2018, tendo por 
base os pressupostos iniciais da pesquisa. A segunda etapa, exploração do material, procurou realizar uma operação classificatória que teve como objetivo alcançar o núcleo de entendimento do texto no que concerne à categoria demografia e obtiveram-se então as caracterizações dos dados. A terceira etapa "Tratamento dos Resultados obtidos e Interpretação do estado bruto dos documentos estudados" foi organizado em figuras e quadros referentes as principais reflexões demográficas de cada região/país. Recorreu-se a interpretação desses dados, considerando os vários períodos censitários que foram ampliados a medida em que se esgotavam informações dos censos atuais dos dois países. As informações que foram pertinentes sofreram um processo de tabulação e análise interpretativa dos dados (CRESWELL, 2010), que são apresentados no presente artigo. Com base na interpretação dessas informações, foi possível produzir dados e refletir sobre as duas realidades distintas.

\section{Apresentação e discussão dos resultados}

\subsection{Dados demográficos: Portugal e Brasil}

Para a compreensão de como se organizam os dados censitários de Portugal e do Brasil, é preciso, primeiro, entender que ambos os países têm dimensões territoriais e volumes populacionais muito distintos. O censo demográfico é realizado, em ambos os países, a cada 10 anos, contudo, Portugal realiza a contagem no primeiro ano da década - último censo foi em 2011 - enquanto o Brasil faz o recenseamento no ano zero da década, tendo sido seu último censo em 2010.

Por se tratar de um país da União Europeia (UE), Portugal tem o seu território geográfico com aproximadamente $92.212 \mathrm{~km}^{2}$. É organizado pela Nomenclatura das Unidades Territoriais para fins estatísticos (NUTS), criada pelo Serviço de Estatística das Comunidades Europeias (Eurostat), em cooperação com os Institutos Nacionais de Estatística dos diferentes países da UE para efeitos de análise estatística de dados, com base numa divisão coerente e estruturada do território econômico comunitário. A NUTS foi instituída pela primeira vez em Portugal pela Resolução de Conselho de Ministros n 34/1986, na sequência da adesão de Portugal à Comunidade Econômica Europeia, com a publicação do Regulamento (CE) n ${ }^{\circ}$ 1.059/2003, do Parlamento Europeu e do Conselho, de 26 de maio (INE, 2015). A NUTS (Figura 1) é formada por três níveis hierárquicos, na medida em que subdivide cada Estado-membro em três níveis: NUTS I, NUTS II e NUTS III. O segundo e terceiro níveis são, respectivamente, subdivisões do primeiro nível.

A NUTS I portuguesa é constituída por três unidades, correspondentes ao território do continente e das regiões Autônomas dos Açores e da Madeira; a NUTS II é composta por sete unidades, das quais cinco ficam no continente, além dos territórios das Regiões Autônomas do Açores e da Madeira; a NUTS 
III é formada por 25 unidades - 23 delas localizam-se no continente e duas correspondem às Regiões Autônomas do Açores e da Madeira (INE, 2015), conforme se observe no quadro 1. Importante ressaltar que as NUTS são apenas utilizadas para fins estatísticos, pois, administrativamente, o país é dividido em municípios e freguesias.

Figura 1 - Representação Geográfica das NUTS I, II, III em Portugal

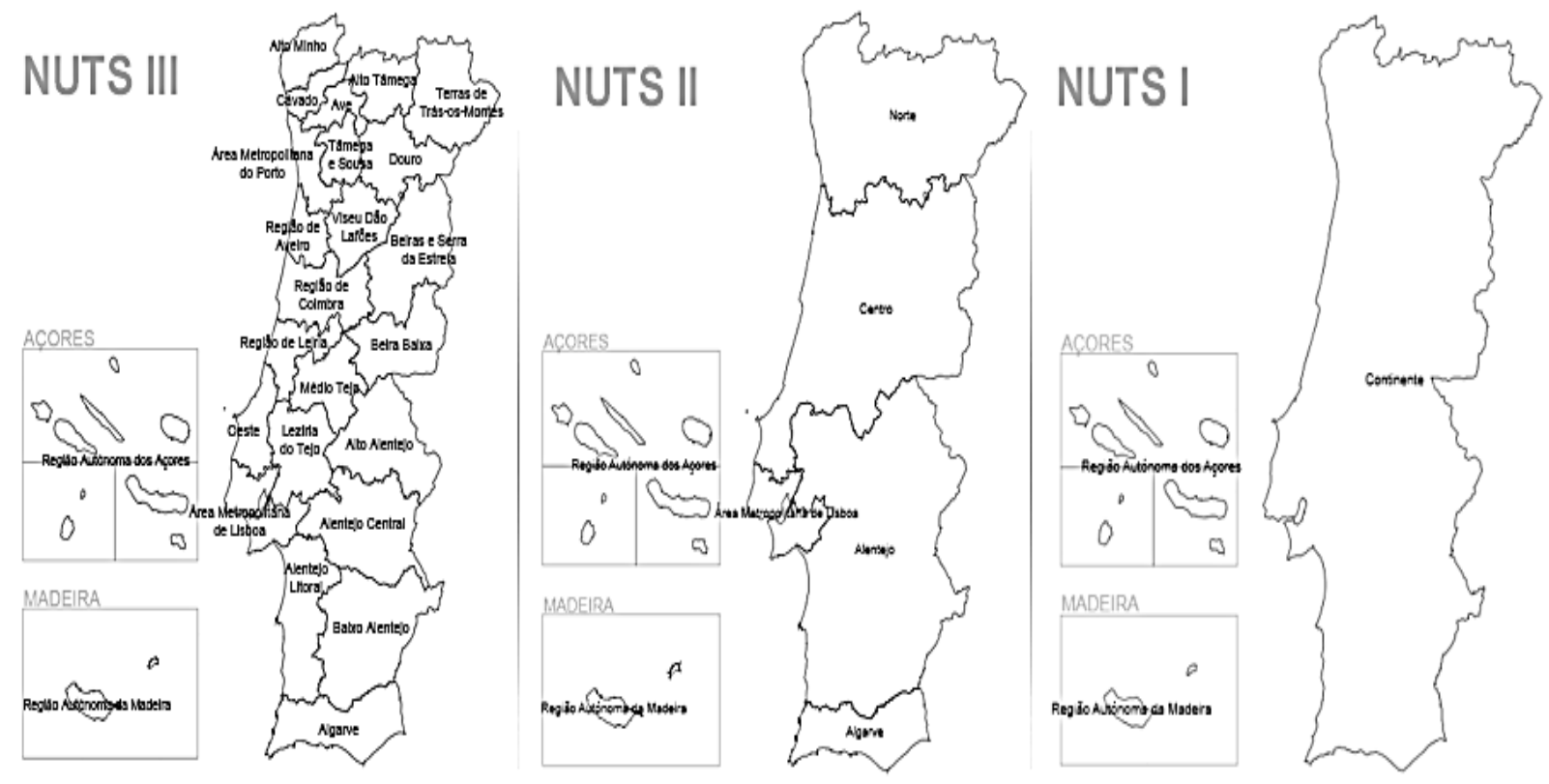

Fonte: Nomenclatura das Unidades Territoriais para fins Estatísticos (INE, 2018). 
Quadro 1- Constituição das NUTS 2018

\begin{tabular}{|c|c|c|}
\hline NUTS I & NUTS II & NUTSIII \\
\hline \multirow{25}{*}{ Continente } & \multirow{8}{*}{ Norte } & Alto Minho \\
\hline & & Cávado \\
\hline & & Ave \\
\hline & & Área Metropolitana do Porto \\
\hline & & Alto Tâmega \\
\hline & & Tâmega e Souza \\
\hline & & Douro \\
\hline & & Terra de Trás-dos-Montes \\
\hline & Algarve & Algarve \\
\hline & \multirow{8}{*}{ Centro } & Oeste \\
\hline & & Região de Aveiro \\
\hline & & Região de Coimbra \\
\hline & & Região de Leiria \\
\hline & & Viseu Dão-Lafões \\
\hline & & Beira Baixa \\
\hline & & Médio Tejo \\
\hline & & Beira da Serra da Estrela \\
\hline & Área Metropolitana de Lisboa & Área Metropolitana de Lisboa \\
\hline & \multirow{5}{*}{ Alentejo } & Alentejo Litoral \\
\hline & & \begin{tabular}{|l|} 
Baixo Alentejo \\
\end{tabular} \\
\hline & & Lezíria do Tejo \\
\hline & & Alto Alentejo \\
\hline & & Alentejo Central \\
\hline & Região Autônoma dos Açores & Região Autônoma dos Açores \\
\hline & Região Autônoma da Madeira & Região Autônoma da Madeira \\
\hline
\end{tabular}

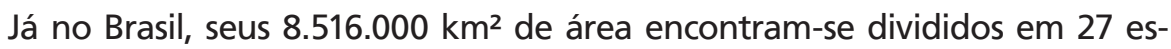
tados, com 5.565 municípios, tendo a região Sul do país 1.188 municípios (IBGE, 2010). A Figura 2 demonstra apenas a divisão territorial por estados, já que, devido à grande quantidade de municípios, seria contrassenso representá-los em figuras:

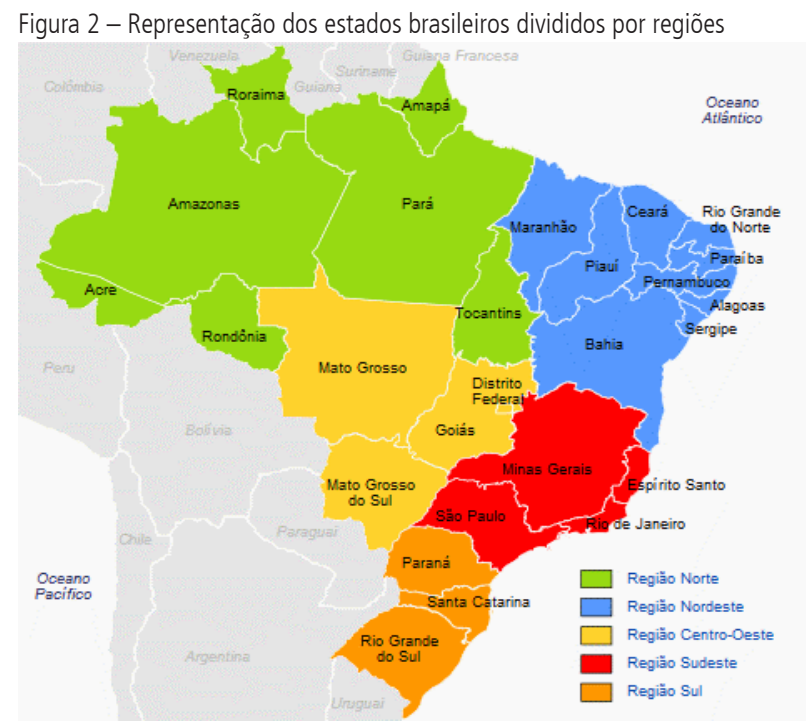

Fonte: IBGE (2010, p. 32). 
Portugal, em termos populacionais, não tem apresentado um crescimento significativo, conforme apresenta a Tabela 1. No período de 1950 a 2011, nota-se que houve pouco crescimento no número de habitantes, especialmente no período de 2001 a 2011, com um aumento de pouco mais de 200 mil pessoas vivendo no país. Por sua vez, o Brasil teve crescimento populacional constante, como pode-se observar na Figura 3, desde o primeiro censo até o mais recente.

Observa-se que os 27 estados que compõem o território brasileiro o transformam em um país continental, aproximando-o da extensão territorial da Europa, que é de $10.180 .000 \mathrm{~km}^{2}$ (NEY et al., 2015). Observa-se que há distinções na constituição territorial e na organização administrativa entre esses dois países. Portugal centraliza as decisões sobre o seu território na capital Lisboa e as tomadas de decisão refletem-se em todo o território nacional, resguardando as devidas particularidades territoriais e decisões políticas. No Brasil, a capital administrativa é Brasília, mas as escalas de governança são descentralizadas em âmbito estadual e municipal (ABRUCIO, 2007).

Tabela 1 - População residente em Portugal

\begin{tabular}{ll}
\hline Recenseamento & Total da população \\
\hline $\mathbf{1 9 5 0}$ & 8.441 .312 \\
$\mathbf{1 9 6 0}$ & 8.889 .392 \\
$\mathbf{1 9 7 0}$ & 8.663 .252 \\
$\mathbf{1 9 8 1}$ & 9.833 .014 \\
$\mathbf{1 9 9 1}$ & 9.867 .147 \\
$\mathbf{2 0 0 1}$ & 10.356 .117 \\
$\mathbf{2 0 1 1}$ & 10.562 .178 \\
\hline
\end{tabular}

Figura 3 - Evolução do crescimento da população brasileira de 1872 a 2010

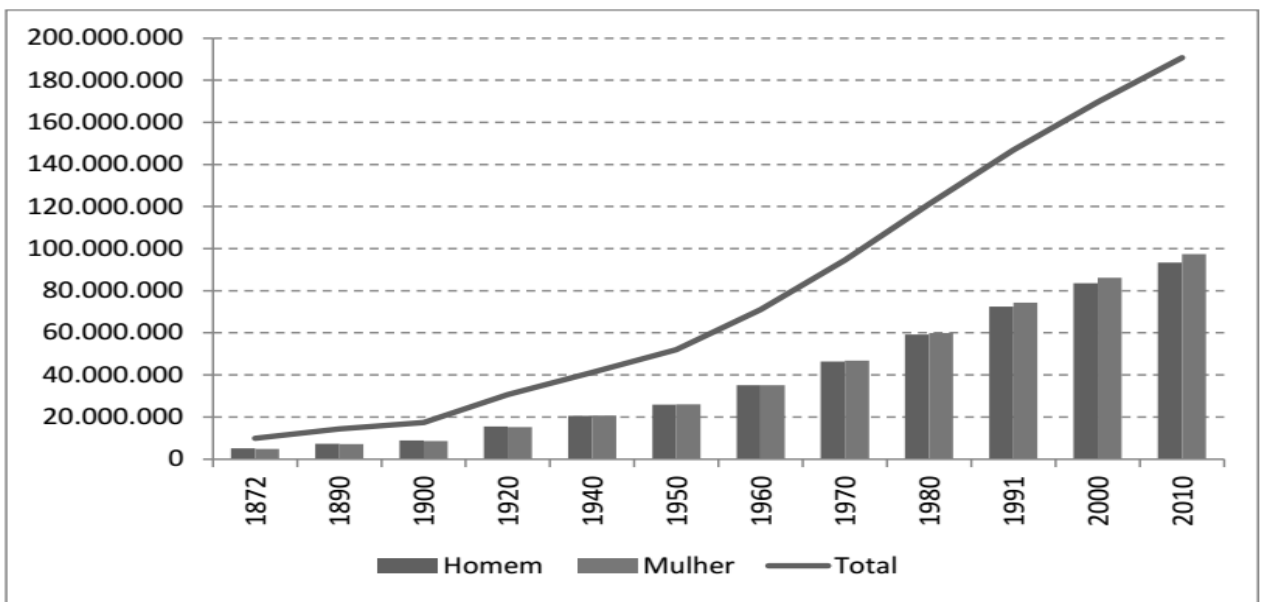

Fonte: Instituto Brasileiro de Geografia e Estatística - IBGE, Censos demográficos (2012).

Destaca-se que desde o primeiro recenseamento brasileiro a população já atingiu 9.930.478 habitantes (IBGE, 2010), um número muito próximo do to- 
tal de habitantes portugueses atual, que é de 10.562 .178 pessoas (INE, 2011). O crescimento populacional no Brasil foi contínuo e progressivo, e, desde o Censo 2000 , a população cresceu $12,3 \%$, isto é, 21 milhões a mais de brasileiros, o equivalente ao dobro da população de Portugal. Em relação ao último censo, a população brasileira era de 190,7 milhões (IBGE, 2010).

\subsection{O envelhecimento Populacional e o Desenvolvimento Regional}

Em Portugal, durante os sessenta anos entre 1950 e 2011, verifica-se que houve um crescimento no envelhecimento da população como resultado de diferentes dinâmicas e circunstâncias (REMOALDO, 2005). Essa alteração torna-se evidente na forma das pirâmides, que espelham a transformação das características da população portuguesa, numa clara inversão da pirâmide de idades (Figura 4).

Figura 4 - População portuguesa em 1950 e em 2011
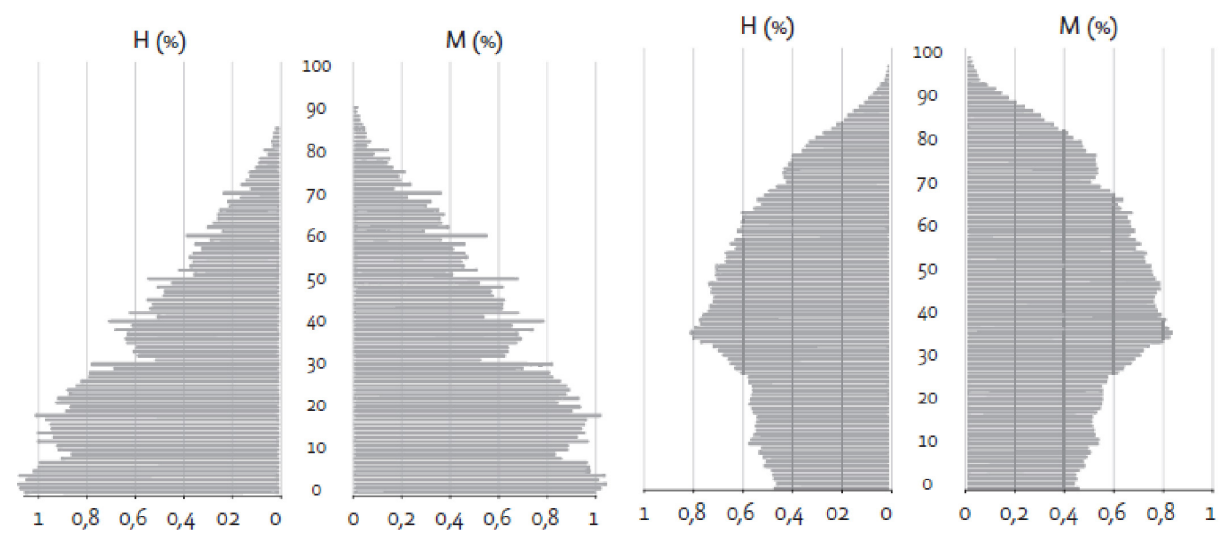

Fonte: INE. Recenseamento da população em 1950 e 2011.

As melhorias das condições de vida, do bem-estar, da área da saúde, das assistências refletiram-se na diminuição da mortalidade e, consequentemente, na esperança de vida. Esses efeitos são visíveis no alargamento do topo da pirâmide e relativo aumento progressivo da população idosa (INE, 2011).

Por sua vez, a diminuição da base traduz os efeitos da diminuição da fecundidade na dinâmica populacional. Esses resultados integram o processo de transição demográfica, que tem sido particularmente discutido, sobretudo em contextos de baixa fecundidade (REHER, 2004, 2007). A forma como esse processo aconteceu, aliado à diminuição da mortalidade e da fecundidade, reflete e explica as transformações ocorridas nas estruturas populacionais, as quais devem ser associadas também aos efeitos das migrações (emigração e imigração). Quanto à proporcionalidade de idosos identificados pelas regiões da NUTS II, verifica-se que o aumento de idosos também é gradativo e constante, como se pode observar na Tabela 2. 
MIRIAM CABRERA CORVELO DELBONI, SILVIA VIRGINIA COUTINHO AREOSA, JULIANA ROHDE, ALINE SARTURI PONTE, NATHALIE DA COSTA NASCIMENTO, ANDREISI CARBONE ANVERSA

Tabela 2 - Proporção de idosos no país e NUTS II de 1950 a 2011

\begin{tabular}{llllllll}
\hline Portugal/NUTS II & $\begin{array}{l}\mathbf{1 9 5 0} \\
(\%)\end{array}$ & $\begin{array}{l}\mathbf{1 9 6 0} \\
(\%)\end{array}$ & $\begin{array}{l}\mathbf{1 9 7 0} \\
(\%)\end{array}$ & $\begin{array}{l}\mathbf{1 9 8 1} \\
(\%)\end{array}$ & $\begin{array}{l}\mathbf{1 9 9 1} \\
(\%)\end{array}$ & $\begin{array}{l}\mathbf{2 0 0 1} \\
(\%)\end{array}$ & $\begin{array}{l}\mathbf{2 0 1 1} \\
(\%)\end{array}$ \\
\hline Portugal & 7,0 & 8,0 & 9,7 & 11,4 & 13,6 & 16,4 & 19,0 \\
Norte & 6,3 & 6,9 & 8,2 & 9,8 & 11,4 & 14,0 & 17,1 \\
Centro & 8,0 & 9,2 & 11,6 & 13,8 & 16,5 & 19,4 & 22,4 \\
Lisboa & 6,6 & 7,9 & 8,7 & 9,7 & 12,3 & 15,4 & 18,2 \\
Alentejo & 6,8 & 8,2 & 11,7 & 15,4 & 18,6 & 22,3 & 24,2 \\
Algarve & 8,7 & 10,5 & 13,9 & 15,8 & 17,3 & 18,6 & 19,5 \\
Região Autónoma dos Açores & 6,7 & 6,7 & 8,8 & 11,3 & 12,5 & 13,0 & 13,1 \\
Regiáo Autónoma da Madeira & 5,7 & 6,5 & 8,2 & 10,5 & 11,6 & 13,7 & 14,9 \\
\hline
\end{tabular}

Fonte: INE. Recenseamentos da População Portuguesa 1950 e 2011, s.p., em 2012.

Tratando-se das NUTS III (Tabela 3), a evolução da proporção de idosos foi sempre crescente de 1950 a 2011, no entanto, analisa-la estabelecer alguns pontos importantes para a compreensão da configuração do envelhecimento da população portuguesa. Embora as NUTS II tenham demonstrado que, em 1950, estivesse em Algarve a proporção de idosos mais expressiva, quando se consideram as NUTS III, é possível perceber que isso ocorre com Pinhal Interior Norte, Pinhal Interior Sul, Serra da Estrela, Algarve, Dão-Lafões, Minho Lima, Baixo Vouga, Baixo Mondego, Médio Tejo e Beira Interior Sul, que apresentam as porcentagens de idosos mais elevadas (INE, 2011). Isso significa que são as NUTS do Interior Centro as que registram o envelhecimento mais intenso, além de Algarve, Minho Lima e NUTS do Centro, duas das quais litorais (Baixo Vouga e Baixo Mondego) e Médio Tejo (da NUTS II Alentejo).

Tabela 3 - Proporção de idosos no país e NUTS III de 1950 a 2011

\begin{tabular}{|c|c|c|c|c|c|c|c|}
\hline Portugal e NUTS III & $\begin{array}{l}1950 \\
(\%) \\
\end{array}$ & $\begin{array}{l}1960 \\
(\%)\end{array}$ & $\begin{array}{l}1970 \\
(\%) \\
\end{array}$ & $\begin{array}{l}1981 \\
(\%)\end{array}$ & $\begin{array}{l}1991 \\
(\%)\end{array}$ & $\begin{array}{l}2001 \\
(\%) \\
\end{array}$ & $\begin{array}{l}2011 \\
(\%)\end{array}$ \\
\hline Portugal & 7,0 & 8,0 & 9,7 & 11,4 & 13,6 & 16,4 & 19,0 \\
\hline Minho Lima & 8,2 & 9,6 & 11,3 & 14,0 & 16,7 & 20,0 & 23,1 \\
\hline Cávado & 6,4 & 7,0 & 7,5 & 8,7 & 9,8 & 11,8 & 14,3 \\
\hline Ave & 5,4 & 5,7 & 6,9 & 8,1 & 9,1 & 11,4 & 14,8 \\
\hline Grande Porto & 5,7 & 6,5 & 7,6 & 8,9 & 10,6 & 13,1 & 16,6 \\
\hline Tâmega & 6,5 & 7,1 & 7,7 & 9,0 & 10,0 & 11,8 & 14,1 \\
\hline Entre Douro e Vouga & 6,8 & 7,0 & 7,6 & 8,8 & 10,3 & 12,6 & 16,4 \\
\hline Douro & 6,6 & 7,4 & 9,9 & 12,6 & 15,6 & 20,0 & 23,2 \\
\hline Alto Trás-os-Montes & 6,1 & 6,4 & 9,5 & 12,8 & 17,3 & 22,7 & 27,9 \\
\hline Baixo Vouga & 8,2 & 8,6 & 9,7 & 11,1 & 12,9 & 15,6 & 18,8 \\
\hline Baixo Mondego & 8,2 & 9,1 & 11,0 & 12,8 & 15,2 & 18,7 & 22,1 \\
\hline Pinhal Litoral & 6,0 & 6,9 & 8,1 & 10,0 & 12,8 & 15,9 & 19,3 \\
\hline Pinhal Interior Norte & 10,4 & 12,5 & 15,9 & 18,5 & 21,5 & 23,6 & 26,1 \\
\hline Dão-Lafões & 8,3 & 9,4 & 11,6 & 14,1 & 16,7 & 19,7 & 23,3 \\
\hline Pinhal Interior Sul & 9,1 & 11,1 & 14,9 & 20,0 & 25,6 & 31,0 & 33,6 \\
\hline Serra da Estrela & 9,1 & 10,6 & 13,2 & 16,4 & 19,8 & 23,9 & 28,8 \\
\hline Interior Norte & 7,5 & 9,1 & 13,6 & 18,1 & 21,7 & 25,4 & 28,6 \\
\hline Beira Interior Sul & 7,7 & 10,0 & 15,4 & 20,5 & 24,0 & 27,5 & 28,7 \\
\hline
\end{tabular}


DEMOGRAFIA E O ENVELHECIMENTO EM PORTUGAL E NO BRASIL: REFLEXÕES PARA O DESENVOLVIMENTO REGIONAL

\begin{tabular}{llllllll} 
Cova da Beira & 6,9 & 8,0 & 11,5 & 15,3 & 18,0 & 21,4 & 25,2 \\
Oeste & 7,1 & 8,4 & 10,3 & 12,2 & 15,0 & 17,9 & 20,1 \\
Médio Tejo & 8,1 & 9,7 & 12,0 & 14,5 & 17,4 & 20,9 & 23,9 \\
Grande Lisboa & 6,9 & 8,3 & 9,2 & 10,2 & 12,8 & 15,8 & 18,3 \\
Península de Setúbal & 5,4 & 6,2 & 6,8 & 8,2 & 10,9 & 14,3 & 18,0 \\
Alentejo Litoral & 4,8 & 6,2 & 9,9 & 14,0 & 16,8 & 21,6 & 23,9 \\
Alto Alentejo & 7,5 & 9,4 & 13,3 & 18,3 & 21,9 & 26,0 & 27,3 \\
Alentejo Central & 6,6 & 7,8 & 11,0 & 15,0 & 18,4 & 22,4 & 24,4 \\
Baixo Alentejo & 6,6 & 7,8 & 12,0 & 16,9 & 20,3 & 24,0 & 25,2 \\
Lezíria do Tejo & 7,6 & 9,3 & 11,7 & 13,4 & 16,7 & 19,8 & 22,0 \\
Algarve & 8,7 & 10,5 & 13,9 & 15,8 & 17,3 & 18,6 & 19,5 \\
Região Autónoma dos Açores & 6,7 & 6,7 & 8,8 & 11,3 & 12,5 & 13,0 & 13,1 \\
Região Autónoma da Madeira & 5,7 & 6,5 & 8,2 & 10,5 & 11,6 & 13,7 & 14,9 \\
\hline Fonte:INE. Recns,
\end{tabular}

Fonte: INE. Recenseamentos da População Portuguesa, 2011.

Bandeira (2000), em sua análise, observou que há algumas sub-regiões, entre elas o Cávado, que surge com um crescimento contínuo na percentagem de idosos e que, no último censo, atingiu os $14 \%$ de idosos, contudo, em outras áreas, essas proporcionalidades oscilam bastante. $O$ autor destaca ainda, na década de 2000 a 2010, a enorme porcentagem de idosos em algumas regiões do interior de Portugal, como Pinhal Interior Sul, com 33,6\%, Serra da Estrela, com 28,8\%; Beira Interior Sul, com 28,7\%, Interior Norte, com 28,6\%, e Trás os Montes, com 27,9\% de idosos. Esses altíssimos índices de proporcionalidade de idosos na população de Portugal, principalmente nas regiões do interior do país, causam extrema preocupação aos gestores de políticas públicas para essa população (BANDEIRA, 2000). Por outro lado, nota-se, na Tabela 4, que, embora entre 1950 e 2011 a população total tenha aumentado, a de jovens diminuiu.

Tabela 4 - População portuguesa segundo os grupos de idades entre 1950 e 2011

\begin{tabular}{lllllll}
\hline $\begin{array}{l}\text { Recensea- } \\
\text { mentos }\end{array}$ & $\mathbf{0 - 1 4}$ & $\mathbf{1 5 - 2 4}$ & $\mathbf{2 5 - 6 4}$ & $\begin{array}{l}\mathbf{6 5} \mathrm{e} \\
\text { mais anos }\end{array}$ & $\begin{array}{l}\mathbf{7 5} \text { e } \\
\text { mais anos }\end{array}$ & Total país \\
\hline $\mathbf{1 9 5 0}$ & 2.488 .085 & 1.572 .667 & 3.791 .045 & 589.515 & 688.910 & 8.441 .312 \\
$\mathbf{1 9 6 0}$ & 2.591 .955 & 1.452 .429 & 4.136 .439 & 708.569 & 238.121 & 8.889 .392 \\
$\mathbf{1 9 7 0}$ & 2.451 .850 & 1.358 .940 & 3.967 .575 & 832.760 & 272.720 & 8.663 .252 \\
$\mathbf{1 9 8 1}$ & 2.508 .673 & 1.628 .059 & 4.570 .824 & 1.125 .458 & 384.812 & 9.833 .014 \\
$\mathbf{1 9 9 1}$ & 1.972 .403 & 1.610 .836 & 4.941 .164 & 1.342 .744 & 527.948 & 9.867 .147 \\
$\mathbf{2 0 0 1}$ & 1.656 .602 & 1.479 .587 & 5.526 .435 & 1.693 .493 & 701.366 & 10.356 .117 \\
$\mathbf{2 0 1 1}$ & 1.572 .329 & 1.147 .315 & 5.832 .470 & 2.010 .064 & 961.925 & 10.562 .178 \\
\hline
\end{tabular}

Fonte: INE. Recenseamentos da população portuguesa, 2011.

Entre os indivíduos de 0 a 14 anos e de 15 a 24 anos, verificou-se um decréscimo constante, com poucas oscilações. A população de 25 a 64 anos registrou um acréscimo significativo, levando à projeção de que somarão mais idosos nas próximas décadas. Entretanto, foi no grupo dos idosos, acima de 65 anos e mais, que o acréscimo populacional foi mais intenso. 
Na Tabela 5, pode-se observar com mais clareza o que significa esse crescimento discrepante entre a população que envelhece e o número de crianças e jovens que decai na população portuguesa em termos de percentuais.

Tabela 5- Grupos funcionais entre 1950 e 2011 (em percentuais \%)

\begin{tabular}{llll}
\hline Recenseamentos & $\mathbf{0 - 1 4}$ & $\mathbf{1 5 - 6 4}$ & $\mathbf{6 5 +}$ \\
\hline $\mathbf{1 9 5 0}$ & 29,5 & 63,5 & 7,0 \\
$\mathbf{1 9 6 0}$ & 29,2 & 62,9 & 8,0 \\
$\mathbf{1 9 7 0}$ & 28,5 & 61,9 & 9,7 \\
$\mathbf{1 9 8 1}$ & 25,5 & 63,0 & 11,4 \\
$\mathbf{1 9 9 1}$ & 20,0 & 66,4 & 13,6 \\
$\mathbf{2 0 0 1}$ & 16,0 & 67,7 & 16,4 \\
$\mathbf{2 0 1 1}$ & 14,9 & 66,1 & 19,0 \\
\hline
\end{tabular}

Fonte: INE. Recenseamentos da população portuguesa, 2011.

A população portuguesa com 65 anos ou mais registra um aumento, passando dos cerca de 7\%, em 1950, para 19\%, em 2011. No entanto, a população em idade ativa, dos 15 aos 64 anos, apresenta flutuações: decresce em 1960 e 1970, comparativamente a 1950, mas registra aumentos percentuais em 1981, 1991, 2001, voltando a apresentar um decréscimo em 2011. Na evolução desse grupo transparecem diversos efeitos: as emigrações intensas dos anos 1960 e 1970, os aumentos populacionais das décadas seguintes (1981, 1991, 2001), bem como os efeitos do processo de envelhecimento ou do retomar da emigração, ocorrido em 2011 (INE, 2012).

Conforme Bandeira (2000), o movimento migratório em Portugal é bastante intenso em todas as épocas por diferentes motivos, sendo a economia seu maior impulsionador. Em todas as décadas, o decréscimo da população portuguesa quase sempre está relacionado à queda de emprego ou à busca de oportunidades de ganhos financeiros em novas oportunidades de mercado. A emigração portuguesa é fortemente identificada na França, em diversos outros países europeus e em suas antigas colônias, como o Brasil e países da África (BANDEIRA, 2000). Contudo, uma das características demarcadas na cultura portuguesa é o saudosismo, que, para Leitão (2015), é um dos motivos que faz com que o português busque sempre voltar a viver em sua terra natal, contribuindo também para o aumento no índice do envelhecimento no país. Esse movimento migratório afeta diretamente os idosos que vivem em Portugal. Em um levantamento em 2015, a Guarda Nacional Republicana, responsável pelo rastreamento anual nos domicílios do país, nomeado de Censos Sênior 2015, encontrou 23.996 idosos que viviam sozinhos, 5.205 que viviam isolados e sem rede de apoio, e outros 6.727 que não estavam enquadrados nas situações anteriores, mas se encontravam em situação de vulnerabilidade, fruto de limitações físicas e mentais (PORTUGAL, 2015).

O Brasil, por sua vez, também vivencia uma significativa transformação em seu perfil populacional, que evidencia um processo de transição demográ- 
fica decorrente do progressivo envelhecimento da população, com aumento relativo e absoluto dos indivíduos com 60 anos ou mais no país, que, segundo se estima, chegará a 32 milhões de pessoas em 2025 (MORAES, 2012). Para Wong e Carvalho (2006), a trajetória demográfica da população brasileira, na primeira metade desse século $\mathrm{XXI}$, tanto em termos de volume quanto à estrutura etária, já está praticamente definida. No final do século XIX e início do século $\mathrm{XX}$, o grande crescimento populacional deveu-se à imigração internacional para suprir a demanda por mão de obra na agricultura e nas indústrias, principalmente na região Sudeste e, particularmente, na região Sul, com as colonizações (BERNARDES, 1997).

Entre os anos 1940 e 1960, houve um declínio significativo da mortalidade, mantendo-se a fecundidade em níveis bastante altos, produzindo, assim, um rápido crescimento populacional, quase estável e jovem. A partir do final da década de 1960, a redução da fecundidade, que se iniciou nos grupos populacionais mais privilegiados e nas regiões mais desenvolvidas, generalizou-se rapidamente e desencadeou o processo de transição da estrutura etária, que levou, provavelmente, a uma nova população quase estável, mas dessa vez com um perfil envelhecido, ritmo de crescimento baixíssimo e, talvez, negativo (WONG; CARVALHO, 2006).

Wong e Carvalho (2006) destacam que a presença de crianças com menos de cinco anos reduziu-se de $15 \%$ para $11 \%$ entre 1970 e 1990. A participação do grupo etário de 5 a 9 anos declinou de 14\% para 12\%. A proporção de crianças nesses dois grupos de idade continuou decrescendo, chegando, em 2010, a tamanhos similares (cada um representava cerca de $9 \%$ da população total). Complementarmente, os grupos mais velhos aumentaram o seu volume de participação: a população de 65 anos ou mais, por exemplo, aumentou de 3,1\%, em 1970, para 5,5\%, em 2000, e 7,4\% em 2010. Pode-se alcançar uma melhor compreensão da trajetória da transição da estrutura etária brasileira no período de 2000 a 2050 ao se considerarem as taxas médias de crescimento anual de grupos etários mais específicos, analisadas na Tabela 6.

Tabela 6- Taxa média anual de crescimento por grupos etários (Brasil, 2000-2011)

\begin{tabular}{lllllll}
\hline Período & Total & \multicolumn{6}{l}{ Grupo Etário (anos) } \\
\hline $\mathbf{2 0 0 0 - 2 0 0 5}$ & 1,45 & $\mathbf{0 - 1 4}$ & $\mathbf{1 5 - 2 4}$ & $\mathbf{2 5 - 6 4}$ & $\mathbf{6 5 - 7 4}$ & $\mathbf{7 5}$ e + \\
$\mathbf{2 0 1 0 - 2 0 1 5}$ & 1,15 & 0,20 & 0,77 & 2,26 & 3,05 & 4,97 \\
$\mathbf{2 0 2 0 - 2 0 2 5}$ & 0,63 & $-0,33$ & $-0,25$ & 1,77 & 3,18 & 4,05 \\
$\mathbf{2 0 3 0 - 2 0 3 5}$ & 0,63 & $-0,33$ & $-0,59$ & 0,70 & 2,34 & 4,54 \\
$\mathbf{2 0 4 5 - 2 0 5 0}$ & 0,28 & $-0,46$ & $-0,36$ & $-0,05$ & 2,48 & 2,38 \\
\hline
\end{tabular}

Os dados indicam que, a partir de 2020, o grupo de 0 a 14 anos, e, de 2030, o grupo de 15 a 24 anos que já apresentavam taxas negativas de crescimento seguem em acentuado aumento dessa perspectiva. A população de 
25 a 64 anos, em que se concentra a força de trabalho, apresentou, até 2005, taxas de crescimento acima de $2 \%$. Seu ritmo de aumento sofrerá forte desaceleração, já no curto prazo, e seu tamanho absoluto deverá se estabilizar por volta de meados do século XX (IBGE, 2010). Os grupos acima de 65 anos aumentarão a taxas positivas e altas durante todo o período e taxas de crescimento superiores a $4 \%$ são esperadas para a população de 75 anos e mais, durante grande parte da primeira metade do presente século. Além disso, demonstra ser muito alto no contingente de idosos, que caracterizará a transição da estrutura etária brasileira durante a primeira metade do presente século.

As consequências de diferentes taxas de crescimento, no tamanho absoluto da população dos vários grupos etários, tornam-se uma questão importante a ser considerada. Apesar de a fecundidade já ter alcançado o nível de reposição e de, certamente, atingir níveis ainda mais baixos, no futuro próximo a população como um todo deverá ainda crescer durante o período analisado. Conviverão grupos etários que diminuirão, em termos absolutos, com outros crescendo rapidamente, a exemplo da população idosa, conforme demonstrado na Figura 5.

Figura 5- População acumulada até a idade indicada (anos), Brasil, 2000-2050

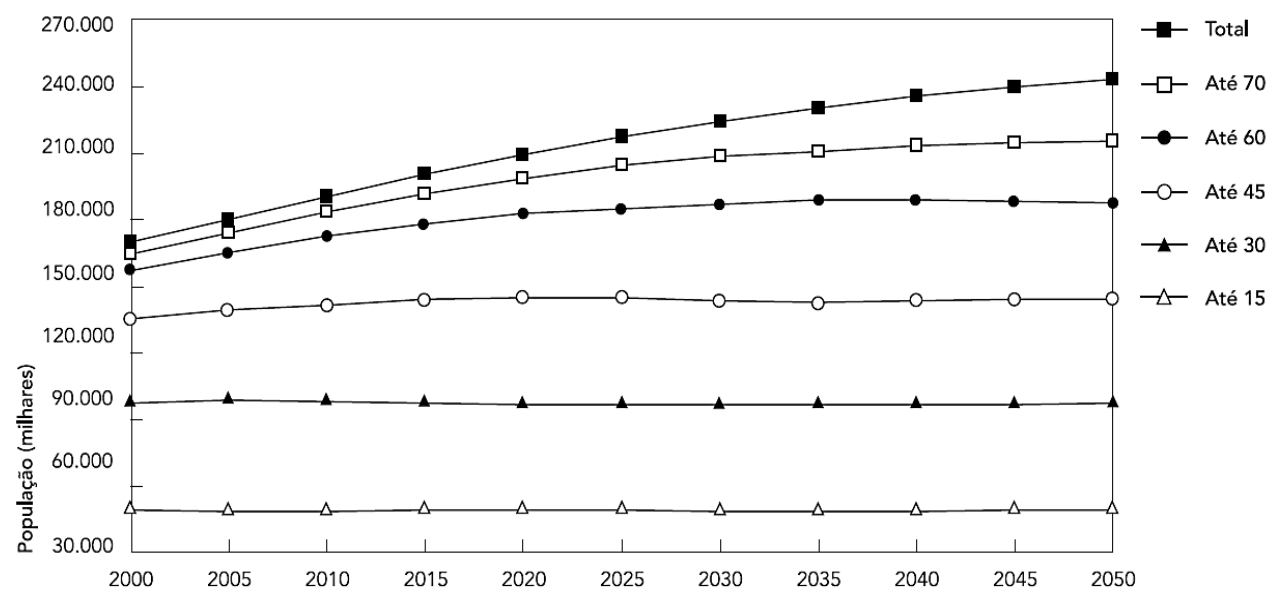

Fonte: IBGE (2010)

As projeções do IBGE (2010), assim como a análise de Carvalho e Wong (2008) e Wong e Carvalho (2006), preveem que, entre 2000 e 2050, 88 milhões de pessoas serão provavelmente adicionadas à população total. Apesar disso, entre os jovens as taxas de crescimento negativas prevalecerão, levando à diminuição no número absoluto desse segmento populacional. Durante o período, o tamanho da população com menos de 15 anos deverá decrescer em algo próximo de cinco milhões. Por sua vez, entre 2000 e 2050, a população total deverá crescer cerca de $50 \%$ e aquela abaixo de 15 anos declinaria em torno de $10 \%$. No último quinquênio analisado, entre 2045 e 2050, a população total do país cresceria, por ano, a uma taxa média de apenas $0,28 \%$ (muito próxima ao 
crescimento nulo) e a abaixo de 15 anos diminuiria a um ritmo de $0,46 \%$ ao ano.

Trata-se de fenômeno registrado pela primeira vez no país e que terá impacto sobre o número absoluto de idosos (65 anos e mais) a partir de 2050. Contudo, a população idosa continuará a crescer em ritmo menor e não haverá declínio na proporção da população brasileira por ela constituída, pois estará ocorrendo, concomitantemente, a diminuição de pessoas em todas as idades abaixo de 45 anos. Isso porque se prevê que o número de nascimentos no país cairá durante todo o período compreendido entre 2010 e 2050. Como já visto, o maior ritmo de crescimento da população idosa levará ao envelhecimento populacional no País. De 3,1\%, em 1970, as pessoas com 65 ou mais anos de idade deverão corresponder, em 2050, a aproximadamente $19 \%$ da população brasileira, sendo que, atualmente, já representam $12,5 \%$ da população total (IBGE, 2018).

Pelos dados apresentados, observa-se que envelhecer foi uma grande conquista da sociedade europeia e também da América do Sul. Entretanto o aumento da duração média de vida para limites considerados inalcançáveis há algumas décadas apresenta-se, hoje, associado às emergências que põem em risco o modo de funcionamento das sociedades modernas. Fortemente ligado à desaceleração do crescimento demográfico, o processo de envelhecimento da população tende a ser associado à fragilização da economia, à limitação da capacidade de empreender e inovar, ao agravamento dos custos indiretos do trabalho e das despesas com os cuidados de saúde (REMOALDO, 2005), e inclusive com a previdência.

Essas são algumas das grandes preocupações com que se debatem, na atualidade, os Estados e as comunidades científicas quando se confrontam com o fenômeno do denominado envelhecimento demográfico, de forma a pensarem na construção de um desenvolvimento regional que contemple as ações junto aos idosos e a população que envelhece. No entanto, há necessidade de uma busca de alternativas positivas para o envelhecimento, para que a cultura do "peso" para a sociedade se transforme em contributo e ação dos idosos. O conhecimento dos aspectos demográficos permite avaliar, além das necessidades, as demandas presentes e futuras de recursos de toda natureza. O conjunto de informações e indicadores gerado pelos estudos demográficos tem especial relevância para a análise das condições de vida da população, acompanhamento e apoio à decisão com relação às políticas públicas, investimentos em saúde e intervenções específicas em áreas críticas (ALCÂNTARA; CAMARANO; GIACOMIM, 2016)

\section{Considerações Finais}

A especificidade da dinâmica populacional tanto de Portugal como do Brasil está associada ao processo histórico de ocupação de seus respectivos territórios. Os dados analisados nos últimos censos dos dois países demonstram 
um crescimento da população de idosos em ambos os países investigados, no caso de Portugal, principalmente, nas regiões do interior do país e no Brasil o acelerado processo de envelhecimento como um fenômeno nacional. A necessidade de gerir ações que visem qualificar tanto o espaço urbano quanto o rural é imperativa, de modo a atender às demandas populacionais atuais e das futuras gerações em ambos os países. Urge a necessidade de se definir estratégicas políticas específicas para essas populações que envelhecem.

Logo, os dados aqui apresentados remetem a reflexão sobre os impactos desse aumento da longevidade e do número de idosos na melhoria e na oferta de cuidados em saúde para a população que envelhece e também a respeito da sustentação econômica dos serviços ofertados em cada região, para além da previdenciária, amplamente debatida no Brasil. Pode-se observar que, apesar das diferentes formas de se organizarem os censos demográficos nos países em estudo, ambos demonstram que o envelhecimento é uma realidade e com a tendência de alcançar índices cada vez maiores nas próximas décadas. Portanto, as análises dos dados censitários aqui demonstrados podem ser utilizadas para a produção de políticas de atenção à saúde e são fundamentais por identificarem a necessidade da manutenção das políticas sociais de proteção ao idoso, visto que são elas que podem auxiliar nas transformações de territórios e na proteção das pessoas que envelhecem.

\section{Referências bibliográficas}

ABRUCIO, F. L. Trajetória recente da gestão pública brasileira: um balanço crítico e a renovação da agenda de reformas. Revista de Administração Pública [online], v. 41, p. 67-86, 2007.

ALCÂNTARA, A. O.; CAMARANO, A. A.; GIACOMIM, K. C. Política nacional do idoso: velhas e novas questões. Rio de Janeiro: Ipea, p.615, 2016.

AREOSA, S. V. C. Envelhecimento humano: realidade familiar e convívio social de idosos do Rio Grande do Sul (Brasil) e da Catalunha (Espanha). Porto Alegre:

EDIPUCRS, 2012.

BANDEIRA, M. S. de M. D. Diogo de Sousa, o urbanista: leituras e texturas de uma cidade refundada. Reposutoriun, Universidade do Minho, Braga, Portugal, 2000.

BECKER, W. Teaching economics in the 21st century. Journal of Economic Perspectives, v. 14, p. 109-119, 2000.

BERNARDES, N. Bases geográficas do povoamento do estado do Rio Grande do Sul. ljuí: Editora Unijuí, 1997.

BERNARDES, F. M. A. Conselho de representação: espaço para os idosos se organizarem na defesa de seus direitos. Revista Kairós, São Paulo, v. 10, n² 2, p. 107-121, dez. 2007.

CARVALHO, J. A. M.; WONG, L. R. A transição da estrutura etária da população brasileira na primeira metade do século XXI. Cadernos de Saúde Pública, Rio de Janeiro, n²4, v. 3, p. 597-605, mar. 2008.

CARVALHO, F. P. A. Fontes das informações para o estudo da mortalidade. In: Ervatti LR, Borges GM, Jardim AP. Mudança demográfica no Brasil no início do século XXI. Subsídios para as projeções da população. Rio de Janeiro, IBGE, 2015. Disponível em: < https://biblioteca.ibge.gov.br/visualizacao/livros/ liv93322.pdf>. Acesso em: 16 abr. 2019.

CRESWELL, J.W. Projeto de Pesquisa: métodos qualitativo, quantitativo e misto. Porto Alegre: Bookman. 2010

GARDNER, P. J. Envelhecimento saudável: uma revisão das pesquisas em Língua Inglesa. Revista Movimento, Porto 
DEMOGRAFIA E O ENVELHECIMENTO EM PORTUGAL E NO BRASIL: REFLEXÕES PARA O DESENVOLVIMENTO REGIONAL

Alegre, v. 12, n² 2, p. 69-92, maio./ago. 2006.

IBGE - Instituto Brasileiro de Geografia e Estatística. Infográficos: evolução populacional e pirâmide etária, 2012. Disponível em: http://cidades.ibge.gov.br/painel/ populacao.php. Acesso em: 2 ago. 2018.

Censo Demográfico 2010. Características gerais da população, religião e pessoas com deficiência. Censo demográfico., Rio de Janeiro, p.1-215, 2010. ISSN 0104-3145. Disponível em: <https://biblioteca.ibge.gov.br/visualizacao/ periodicos/94/cd_2010_religiao_deficiencia.pdf>. Acesso em: 13. jun. 2018.

Censo Demográfico 2000. Características gerais da população. Resultados da amostra. Disponível em: https://biblioteca.ibge.gov.br/visualizacao/periodicos/83/ cd_2000_caracteristicas_populacao_amostra.pdf>. Acesso em: 13.jun. 2018.

Infográficos: evolução populacional e pirâmide etária. Disponível em: <http:// cidades.ibge.gov.br/painel/populacao.php>. Acesso em: 2 ago. 2018.

INE - Instituto Nacional de Estatística. Recenseamento 2012. Disponível em: http://www.ine. st/2012.html. Acesso em: ago. 2018.

Censos 2011. Disponível em: http://censos.ine.pt/xportal/xmain

Nuts 2013: as novas unidades territoriais para fins estatísticos. Lisboa, 2015.

Nuts 2013: as novas unidades territoriais para fins estatísticos. Lisboa, 2015. Disponível em: http://www.poci-compete2020.pt/admin/images/NUTS2013_(1). pdf.

KIST, R. B. B.; AEROSA, S. V. C. Envelhecer na perspectiva do envelhecimento satisfatório: o caso dos idosos do Vale do Rio Pardo. REDES - Revista do Desenvolvimento Regional, Santa Cruz do Sul, v. 19, ed. especial, p. 1-184, 2014.

LEITÃO, A. M. As lutas contra o salazarismo no Brasil: Estudos a serem aprofundados. Projeto História, São Paulo, nº 53, p. 275-285, maio.-ago. 2015.

MINAYO, M. C. Ciência, técnica e arte: o desafio da pesquisa social. In: (Org.). Pesquisa social: teoria, método e criatividade. 21. ed. Petrópolis: Vozes, p. 9- 29, 2002.

MORAES, E. N. de. Atenção à saúde do idoso: aspectos conceituais. São Paulo:

OPAS, 102 p., 2012.

NEY, M. S.; PIERANTONI, C. R.; LAPÃO, L. V. Sistemas de avaliação profissional e contratualização da gestão na Atenção Primária à Saúde em Portugal. SAÚDE DEBATE, Rio de Janeiro, v. 39, n 104, p. 43-55, jan.-mar. 2015.

ONU - Organização das Nações Unidas. Plano de ação internacional para o envelhecimento. Trad. de Arlene Santos. Brasília: Secretaria Especial dos Direitos Humanos, 2002.

Population by Age Groups - Both Sexes. World Population Prospects 2017. Disponível em: <https://population.un.org/wpp/Download/Standard/Population/> Acesso em: 13.jun. 2018.

PORTUGAL. Direção-Geral da Saúde. A saúde dos portugueses. Perspectiva 2015. Lisboa: Direção-Geral da Saúde, 2015. Disponível em: https://www.dgs.pt/estatisticasdesaude/estatisticas-de-saude/publicacoes/a-saude-dos-portugueses-perspetiva2015. aspx. Acesso em: jul. 2018.

REHER, D. S. Rumo a um declínio demográfico de longo prazo: discussão de questões relevantes. Revista Européia de População, v. 23, n II, p. 189- 207, 2007.

. A transição demográfica revisitada como um processo global. População, Espaço e Lugar, v. 10, p. 19-41, 2004.

REMOALDO, P. C. A. A Geografia da Saúde portuguesa-sonhos e realidades. Territoris 
MIRIAM CABRERA CORVELO DELBONI, SILVIA VIRGINIA COUTINHO AREOSA, JULIANA ROHDE, ALINE SARTURI PONTE, NATHALIE DA COSTA NASCIMENTO, ANDREISI CARBONE ANVERSA

Universitat de les Illes Balears, n 5, p. 33-48, 2005.

SEN, A. Desenvolvimento como liberdade. São Paulo: Companhia das Letras, 2000.

SILVEIRA, R. L. L. da; FELIPPI, Â. C. T.; CAMPOS, H. Á. O observatório do Desenvolvimento Regional: a construção de uma rede de pesquisa e extensão sobre a dinâmica do desenvolvimento regional brasileiro. In: SILVEIRA, R. L. L. da. (Org.). Observando o desenvolvimento regional brasileiro: processos, políticas e planejamento. Santa Cruz do Sul: EDUNISC, p. 11-31, 2013.

WONG, L. L. R.; CARVALHO, J. A. O rápido processo de envelhecimento populacional do Brasil: sérios desafios para as políticas públicas. Revista Brasileira de Estudos Populacionais, v. 23, n 1, p. 5-26, 2006. 